\title{
Do Humans Prefer Cognitive Effort Over Doing Nothing?
}

\author{
Raymond $\mathrm{Wu}^{1,2}$, Amanda M. Ferguson ${ }^{3}$, and Michael Inzlicht ${ }^{2,4}$ \\ ${ }^{1}$ Department of Psychology, University of British Columbia \\ ${ }^{2}$ Department of Psychology, University of Toronto \\ ${ }^{3}$ Graduate Department of Psychological Clinical Science, University of Toronto \\ ${ }^{4}$ Rotman School of Management, University of Toronto
}

In press at Journal of Experimental Psychology: General

\begin{abstract}
Author Note
Raymond WuiD https://orcid.org/0000-0003-4750-0806

Amanda M. Ferguson (D) https://orcid.org/0000-0002-5451-9209

Michael Inzlicht (D) https://orcid.org/0000-0001-9297-6497

We have no conflicts of interest to disclose.

Correspondence concerning this article should be addressed to Raymond $\mathrm{Wu}$, University of British Columbia, 6398 University Blvd, Vancouver, BC V6T 1Z4. Email: rwu@psych.ubc.ca Data, codebook, materials, and supplemental materials: https://osf.io/s4wv9/

Preregistrations: https://aspredicted.org/KXV_6SH; https://aspredicted.org/2MG_3MF; https://aspredicted.org/YBR_5MP
\end{abstract}




\begin{abstract}
Humans and other animals find mental (and physical) effort aversive and have the fundamental drive to avoid it. However, doing nothing is also aversive. Here, we ask whether people choose to avoid effort when the alternative is to do nothing at all. Across twelve studies, participants completed variants of the demand selection task, in which they repeatedly selected between a cognitively effortful task (e.g., simple addition, Stroop task, symbol-counting task) and a task that required no effort (e.g., doing nothing, watching the computer complete the Stroop, symbolviewing). We then tabulated people's choices. Across our studies and an internal meta-analysis, we found little evidence that people choose to avoid effort (and hints that people sometimes prefer effort) when the alternative was doing nothing. Our findings suggest that doing nothing can be just as costly - if not more costly - than exerting effort.
\end{abstract}

Keywords: cognitive control, boredom, decision making, mental effort, effort avoidance 


\section{Do Humans Prefer Cognitive Effort Over Doing Nothing?}

Humans are regularly required to exert cognitive effort, be it to navigate through a busy street, maintain healthy relationships, or resist doughnuts. Unfortunately, humans (and other animals) seem to be effort misers, tending to minimize effort whenever possible. We find effort aversive and avoid it as a result. However, minimizing effort too much, inevitably leading to doing absolutely nothing, is also aversive. People find themselves at a loss when there is nothing to do. Many of us get bored easily and go to great lengths to avoid it. In such cases, people work hard to escape doing nothing. Boredom and effort are two variables that shape decisions Yet, it is unknown whether the fundamental drive to avoid effort has precedence over the aversiveness of doing nothing. We address this gap by pitting these two drives against one another, asking whether people choose to avoid effort in the face of doing nothing.

\section{Cognitive Effort Avoidance}

People tend to avoid cognitive effort. The law of least effort states that when given equally rewarding options, organisms will learn to choose the least effortful option (Hull, 1943). Indeed, across a wide range of contexts, people reduce expending cognitive effort when given the option to do so (Cameron et al., 2019; Kool et al., 2010; Vogel et al., 2021; Westbrook et al., 2013; Zipf, 1949). Additionally, the avoidance of effort is not merely due to error avoidance, demand characteristics, or stimuli-dependence (Kool et al., 2010; c.f. Dunn et al., 2019). Crucially, even though effort itself can sometimes be rewarding (Inzlicht et al., 2018) and even invigorating (Milyavskaya et al., 2021), effort avoidance only terminates when effort is adequately compensated with rewards (Clay et al., 2022; Kool \& Botvinick, 2014; Devine \& Otto, 2021). 
Expending cognitive effort feels aversive. Given that cognitive control is computationally limited, people cannot meet the demands of all tasks simultaneously and thus must prioritize tasks (Shenhav et al., 2017). The limits of control mean that engaging control for one task forgoes the potential rewards of engaging control for an alternative task. This foregoing of another opportunity (known as opportunity costs) is phenomenologically felt as effort, which is aversive, unpleasant, and costly (Kurzban et al., 2013). Effort, in fact, might feel aversive so as to prevent organisms from exerting control promiscuously, thereby minimizing opportunity costs (Kurzban et al., 2013). While there is not yet consensus as to why effort is aversive (Kool \& Botvinick, 2018), it is aversive, with people and other animals avoiding it as a result.

The typical effort avoidance paradigm compares preferences between cognitive control tasks that vary on level of demand. For instance, people might choose between two variations of the Stroop task, one with more congruent trials (low effort) and one with more incongruent trials (high effort; Schouppe et al., 2014). Here, we ask if people would continue to avoid cognitive effort if the choice is between effort and doing nothing at all. If the options differ only on the effort required and all else is equal, then the law of least effort might suggest so - after all, doing nothing requires little or no effort. But there are reasons to believe that all else will not be equal, leading people to find that doing nothing is equally or even more aversive than effort.

\section{Doing Nothing is Aversive}

Activities that require people to do nothing may be aversive because they inevitably lead to boredom. Humans prefer an optimal level of stimulation (Leuba, 1955). When there is a lack of (or sometimes too much) stimulation, however, it can lead to boredom, which is characterized by a lack of attentional engagement and meaningfulness. Boredom motivates the search for alternative tasks, especially tasks that are personally rewarding (Bench \& Lench, 2013; 
Eastwood et al., 2012; Milyavskaya et al., 2019; Tam et al., 2021; Westgate \& Wilson, 2018). Evidence suggests that, to reduce boredom, people are even willing to explore other costly experiences, such as paying money (Dal Mas \& Wittmann, 2017), looking at disgusting images (Bench \& Lench, 2019), self-administering electric shocks (Nederkoorn et al., 2016; Wilson et al., 2014), breaking rules (Boylan et al., 2021; Wolff et al., 2020), and even acting sadistically (Pfattheicher et al., 2020).

Boredom, however, is functional: it stimulates exploration. Humans (and other animals) face a challenge between exploiting a known reward and exploring the environment for information that can potentially lead to even better rewards. Known as the explore/exploit tradeoff (Cohen et al., 2007), theorists have posited that boredom acts as a signal to start exploring one's environment to gain valuable information (Agrawal et al., 2021; Danckert, 2019; Geana et al., 2016; Klapp, 1986). That is, given that dull tasks typically provide little useful information, boredom might instigate the drive to explore the environment in search of more rewarding tasks, which might involve engaging effort (Petitet et al., 2021). For instance, tasks that provide little valuable information are perceived as more boring and are quicker to be skipped in favor of an alternative task (Geana et al., 2016). At the same time, however, exploration may not always yield any additional information. In such cases, it may be optimal to gradually cease exploration and tolerate boredom instead.

\section{Current Investigation}

The present studies aim to determine whether people will avoid cognitive effort if the alternative is to do nothing. Across twelve studies, participants chose between cognitively effortful tasks and doing nothing. We additionally examined phenomenological ratings of effort and boredom of the tasks along with individual differences in effort preferences and boredom 
proneness. If people truly avoid cognitive effort, they should prefer doing nothing. However, we believe this to be unlikely because doing nothing is also aversive. While we pre-registered some of our studies (https://aspredicted.org/KXV_6SH; https://aspredicted.org/2MG_3MF; https://aspredicted.org/YBR_5MP), given the opposing drives, we did not derive clear directional predictions. We suspected that people would not avoid cognitive effort but were uncertain to what extent they would willingly engage effort to avoid doing nothing.

\section{Methods}

\section{Transparency and Openness}

We report how we determined our sample sizes, all data exclusions, and all measures in our studies. These, along with our data, research materials and codebook, have been made publicly available on the Open Science Framework and can be access at https://osf.io/s4wv9/. Data were analyzed using Statistical Package for the Social Sciences (version 28) and R Studio (version 1.4). Jamovi was used to meta-analyze choices. Although we did not formally preregister all our studies, we preregistered the study design, hypothesis, exclusion criteria, and analysis plan for Studies 2, 9, 11 and 12 (https://aspredicted.org/KXV_6SH; https://aspredicted.org/2MG_3MF; https://aspredicted.org/YBR_5MP)

\section{Participants and Procedure}

Across thirteen studies (including the pilot study), 2,311 participants were recruited from the University of Toronto Scarborough undergraduate student pool and Prolific Academic, an online participant pool. The institutional review board of the authors' university approved the studies. Following a sensitivity analysis, we aimed to include 120 participants in each study, ${ }^{1}$ allowing us to detect a small effect $(d=.26)$ given our within-person design at an alpha of 0.05

\footnotetext{
${ }^{1}$ This did not apply to Study 12. The sample size in Study 12 is low because we ran it in the winter of 2022 when COVID-19 pandemic restrictions prevented us from running a greater number of participants.
} 
and $80 \%$ power (Faul et al., 2007). We over-sampled given our online design. Although exclusion rates (described below) were higher than expected, we pre-registered them in some of our studies (https://aspredicted.org/KXV_6SH; https://aspredicted.org/2MG_3MF; https://aspredicted.org/YBR_5MP), and our sample sizes were still comparable to, and sometimes larger than, previous within-person designs examining effort avoidance (Cameron et al., 2018, Ferguson et al., 2020; Kool et al., 2010; Schouppe et al., 2014). Our final analysis included 1,274 participants $\left(M_{a g e}=25.4, S D_{a g e}=7.8 \text {, female }=53 \%\right)^{2}$. Table S1 shows our collected and final sample sizes, exclusion rates, and demographics across our studies.

Given that our study consisted of doing aversive tasks online, we implemented three exclusion criteria to ensure that we only included participants that followed instructions and were engaged in our studies. First, to ensure that participants read our questions, they were excluded if they failed at least one of two attention checks $(n=218)$. Second, to ensure that participants did not randomly make choices while doing something else, they were excluded if they failed more than $25 \%$ of trials on our effortful but still simple tasks $(n=211)$. Third, at the end of our studies, we asked, "Did you do anything else (e.g., switch to another tab, look at your phone) during this study? Please be honest. You will receive full compensation regardless of your response." Participants were excluded if they admitted to doing something else during the study $(n=780)$. We preregistered this last exclusion criteria because we were concerned that in an uncontrolled online context, participants might check their phones or do something else as a means to escape the aversive feelings that are evoked by doing nothing. While our main analyses were done after exclusions (as we preregistered), we repeated these analyses with varying exclusion criteria

\footnotetext{
${ }^{2}$ While exclusion rates are high, Prolific samples fail attention checks at a rate of over 30\% (Eyal et al., 2021). Given the aversiveness of both effort and boredom, we included extra data quality checks to ensure participants were not simply pressing buttons randomly and/or doing other tasks concurrently.
} 
(including no exclusions) to test the robustness of our results (see our Supplemental Online Materials). Although results vary somewhat across exclusion criteria, our overall conclusionthat people do not avoid effort when the choice is to do nothing - is consistent across analytic choices.

\section{Materials}

\section{Demand Selection Task}

Participants completed variations of the well-established demand selection task (Kool et al., 2010). Figure 1 shows an example illustration of the task. In each trial, two options, in the form of different colored decks, were presented to the left and right of the middle of the screen. Each deck corresponded to a task with or without demand. Participants were instructed that they should try both decks but if one begins to feel preferable then they should choose that deck. In Studies $10-12$, we emphasize this point by explicitly mentioning to participants that "both decks provide us with equally valuable data and are perfectly valid options" and that "you should not feel that we expect you to prefer any one of the decks; both decks are equally acceptable choices." We operationalized demand with the add-3 task in Studies 1 and 2 (Kahneman 1973), the add-1 task in Studies 3 to 5, the add-variety task in Study 6, the Stroop task in Studies 7 to 9 and 12, and symbol-counting task in Studies 10 and 11 (Garavan et al., 2000; Seiler et al., 2022). The deck with no demand (i.e., do-nothing) option presented a white screen with the words "do nothing" in black font in Studies 1 to 7 and 12, a video recording of the Stroop task completing itself automatically in Studies 8 and 9, and a sequence consisting of two different images in Studies 10 and 11. We programmed trials of both decks to last ten seconds each. ${ }^{3}$

\footnotetext{
${ }^{3}$ Although we programmed all decks to be ten seconds, the Stroop task took slightly longer to complete due to the online browser loading time of the trials. Thus, in Study 7 and 12, the Stroop deck took longer to complete than doing nothing, possibly making it more aversive.
} 
The add tasks presented a string of four digits one at a time in a row. Each digit was displayed for one second and then disappeared. After the entire sequence completes, a text box appeared for the remainder of the trial. Participants were required to add the same number (depending on the task variation) to each of the digits and then type the answer in the textbox using the keyboard (e.g., the correct answer for "1 72 9" in the add-3 is 4052). Correct responses corresponded to getting the entire sequence correct. The Stroop task presented color words in different colored font (e.g., "red" in green font). Participants used their mouse cursor to select their response. Response options (blue, yellow, red, green) were displayed in a 2x2 array. Correct responses corresponded to indicating the color of each word. For the symbol-counting task, we used a validated boredom task (Seiler et al., 2022) that presented images but modified it such that participants were required to count the number of each alternating image (benches and umbrellas) that were displayed. The symbol-counting task requires effort as it draws on the shifting and updating aspects of executive function (Garavan et al., 2000). Each image was displayed for $750 \mathrm{~ms}$. After each trial, participants used two sliders to indicate the count of each image type. In the do-nothing version, participants were shown the same two sliders, but the sliders were "greyed out" and asked them to do nothing. To prevent participants from quickly skipping this question, we implemented a seven second wait period before they could submit the page.

\section{Phenomenological Ratings and Individual Differences}

Participants also reported the degree of mental demand and boredom they experienced for each deck after they completed the demand selection task. This was important because the effortful tasks were repetitive and likely boring. Participants answered the following questions about both decks: "How mentally demanding was this deck?" and "How boring was this deck?" 
Responses were made on a slider ranging from 1(Very Low) to 7 (Very High). In each study, participants found the demand deck more mentally demanding and less boring than the donothing deck (see Table 1). In some of our studies, we also included a full set of phenomenological ratings, which included the degree of (1) physical demand, (2) effortfulness, (3) frustration, (4) success, (5) temporal demand, (6) rumination, and (7) meaningfulness. Participants generally found the demand deck to be more physically demanding, more effortful, equally or more frustrating, more difficult, and more meaningful than the do-nothing deck. In only one of three studies where we assessed rumination ("How much did you think about how sad or upset you felt?"), participant reported more rumination when doing nothing (see Table S3 for all ratings).

We also explored three individual difference variables. Participants completed: The need for cognition scale (Cacioppo et al., 1982) which measures individual differences in enjoyment of cognitive effort, the meaningfulness of effort scale (Campbell, Chung, \& Inzlicht, 2022) which measures the degree to which people find pushing themselves to be meaningful, and the boredom proneness scale (Struk et al., 2016) which measures propensity to feel bored. In Studies 10-12, participants also completed the social desirability scale (He et al., 2015) which measures the tendency to respond in ways that are viewed as favorable by other people. Details of scales are in the Online Supplemental.

\section{Results}

\section{Pilot Study}

In a pilot study, we first sought to demonstrate effort avoidance in an online context. Forty-nine online participants made choices between the add-1 and add-3 task. Across 40 trials, participants showed effort avoidance, choosing the add-3 option $38.57 \%(S D=15.77 \%)$ of the 
time, significantly less than chance $(50 \%), t(48)=-5.07, p<.001, d=-.72,95 \%$ CI $[-1.04,-.41]$.

These results suggest that we can readily find strong effort avoidance on demand selection tasks even in an uncontrolled online context.

\section{Studies 1 to 2: High-Demand vs Do-Nothing}

Next, we examined whether people would still avoid the high-demand option if the less demanding option offered no demand rather than low demand. Participants chose between the add-3 task and doing nothing. In Study 1, across 40 trials, results showed that $59.64 \%$ $(S D=19.92 \%)$ of choices were for the add-3 option, which was significantly higher than chance $(50 \%), t(62)=3.84, p<.001, d=.48,95 \%$ CI $[.22, .75]$. See Table 2 for the proportion of choices in each study, as well as sample sizes, and basic inferential statistics. To examine the robustness of this effect, we sought to replicate these findings with 80 trials in Study 2 (pre-registered: https://aspredicted.org/KXV_6SH). Again, participants chose the add-3 option $(M=58.25 \%$, $S D=20.28 \%$ ) significantly more than chance $(50 \%), t(62)=3.23, p=.002, d=.41,95 \%$ CI $[.15$, .67]. As expected, participants did not avoid effort to do nothing. There is a possibility that people do not avoid any task in favor of doing nothing. To test this, in our next set of studies, we lowered the demand of our high-demand option to see whether people will avoid a low-demand option to do nothing.

\section{Studies 3 to 5: Low-Demand vs Do-Nothing}

To test whether people avoid low demand to do nothing, participants chose between the add-1 task and the do-nothing task. Across 25 trials in Study $3(n=123)$ and 40 trials in Study 4 $(n=62)$, participants were indifferent between the add-1 and do-nothing options (see Table 2): Study $3, t(122)=.03, p=.914, \mathrm{~d}=.002,95 \%$ CI [-.17, .19]; Study $4, t(61)=-.30, p=.769, \mathrm{~d}=-.04$, $95 \%$ CI $[-.29, .21]$. In Study 5, participants chose the add-1 option $45.57 \%$ of the time 
$(\mathrm{SD}=21.81 \%)$, significantly less than chance $(50 \%), t(145)=-2.46, p=.015, d=-.20,95 \% \mathrm{CI}[-.37$,

-.04]. Even though the add-1 task required more effort than doing nothing, participants may have been indifferent between them and sometimes avoided the add-1, because the add- 1 task is repetitive, unchallenging, and perhaps boring. In our next study, we examined choices between a task that varied demand level and doing nothing.

\section{Study 6: Varied-Demand vs Do-Nothing}

In Study 6, we minimized repetition within task to increase the distinction between the low and high demand task options. To this end, we created a version of the add task that randomly presents either the add-1, add-2, add-3, or add-4 in each trial. Importantly, the addvariety task still required cognitive effort, which is aversive and often avoided. Across 40 trials, results showed that choices for the add-variety option $(M=55.24 \%, S D=22.41 \%)$ were significantly higher than chance $(50 \%), t(104)=2.40, p=.018, d=.23,95 \%$ CI $[.04, .43]$. These results suggest that varying demand may make effort more interesting and less aversive.

\section{Study 7: Stroop Task vs Do-Nothing}

In our next set of studies, we examined whether participants would avoid the Stroop task to do nothing across 40 trials. In Study 7, conceptually replicating our past findings, participants chose the Stroop task option $(M=54.07 \%, S D=20.55 \%)$ at a proportion no different from chance $(50 \%), t(82)=1.80, p=.075, d=.20,95 \% \mathrm{CI}[-.02, .42]$. These results suggest that our findings that people are not willing to do nothing to avoid effort generalizes to other effortful tasks.

\section{Studies 8 and 9: Stroop Task vs Do-Nothing - Visual Stimulation}

We next aimed to test whether choices were driven by desire for visual stimulation, given that doing nothing lacks stimulation, which is aversive (Raffaelli et al., 2018). In Study 8, participants chose between completing the Stroop task themselves and passively watching the 
computer automatically complete the Stroop task. Replicating our past findings, results showed that participants chose the Stroop task option $63.37 \%(S D=18.48 \%)$ of the time, which was significantly higher than chance $(50 \%), t(123)=8.06, p<.001, d=.72,95 \%$ CI $[.53, .92]$. To bolster these findings, we replicated these results with 100 trials in Study 9 (pre-registered: https://aspredicted.org/KXV_6SH). Again, participants chose the Stroop task option $(M=67.30 \%, S D=23.35)$ significantly more than chance $(50 \%), t(89)=7.03, p<.001, d=.74,95 \%$ CI $[.51, .98]$. These results suggest that our findings that people do not avoid effort to do nothing were not merely due to the desire for visual stimulation.

\section{Studies 10-11: Count versus Do-Nothing - Demand Characteristics}

Participants may have chosen to exert effort instead of do nothing because they felt obliged to so something to earn their pay and thereby reduce cognitive dissonance (Festinger, 1957). Cognitive dissonance refers to the aversiveness of having inconsistent thoughts and behaviors, such that people will adjust one so they become consistent. Given that participants are usually paid to exert effort in online experiments, they may experience cognitive dissonance if they do nothing (knowing they are getting paid), thereby driving them to choose effort. If this is the case, then participants who are paid more should experience a stronger drive to reduce cognitive dissonance, and thus choose effort even more. To test this, we randomly assigned participants to receive relatively higher or lower remuneration ( $£ 3.75$ or $£ 2.50$ in Studies 10 and $£ 7.50$ or $£ 5.00$ in Study 11; https://aspredicted.org/YBR_5MP). ${ }^{4}$ Participants chose between the symbol-counting task or passively viewing the same images. Across 25 trials in Study 10 and 40 trials in Study 11, we found no difference in choices for demand between the high and low

\footnotetext{
${ }^{4}$ After consenting, participants were presented with the following statements: You have been randomly assigned to earn $£ 3.75$ instead of $£ 2$ for completing the study" or (for those in the control condition) "You will earn $£ 2$ for completing the study" in Study 11, and "You have been randomly assigned to earn $£ 7.5$ instead of $£ 5$ for completing the study" or (for those in the control) "You will earn $£ 5$ for completing the study" in Study 12.
} 
remuneration group: Study $10(n=166), t(159)=1.67, p=.098, d=.26,95 \%$ CI [-.05, .56]; Study 12 $(n=161), t(148)=-.26, p=.797, d=-.04,95 \%$ CI $[-.35, .27]$. Thus, we do not find evidence that people sometimes prefer to exert effort because they feel obliged to the experimenter who is paying them.

Choices for the effortful option may have been motivated by desires to maintain a positive reputation. Whereas doing nothing often feels like a "waste of time," exerting effort is viewed by others as moral (Celniker et al., 2022) and thus socially desirable. If choices were influenced by social desirability, then participants who tend to act in more socially desirable ways should make more effort choices to avoid doing nothing. To see if this was the case, we examined the correlations between individual differences in social desirability and effort choices. Results showed that social desirability was not significantly correlated with choosing the symbol counting option in both Study 10, $r=.09,95 \%$ CI [-.07,.24], $p=.261$, and Study $11, r=.13,95 \%$ CI $[-.03, .28], p=.104$, suggesting that effort choices were not strongly motivated by desires to maintain a positive reputation.

Replicating our previous findings, across 25 trials in Study 11, we found that participants chose the symbol-counting task option $(M=59.16 \%, S D=23.79 \%)$ significantly more than chance level, $t(165)=4.96, p<.001, d=.38,95 \%$ CI $[.23, .54]$. In Study 12, we increased the number of trials from 25 to 40, and participants became indifferent between the two options, $t(160)=.51$, $p=.614, d=.04,95 \%$ CI $[-.12, .19]$. In addition to replicating our previous findings that people do not avoid effort when the alternative is doing nothing, these results further suggest that people may be less inclined to use effort for the same task as they complete more trials.

\section{Study 12: Laboratory Replication}


In Study 12, we sought to replicate our findings in a controlled laboratory setting. Across 100 trials, participants chose between doing the Stroop task or doing nothing (same as Study 7 except more trials; https://aspredicted.org/2MG_3MF). With 100 trials, participants chose the Stroop task $40.05 \%$ of the time $(S D=18.72 \%)$, significantly less than chance $(50 \%), t(38)=-3.32$, $p=.002, d=-.53,95 \%$ CI $[-.88,-.20]$. Although these results seem to be inconsistent with our online findings (Study 7), on closer inspection we replicate our findings when we examine the same number of trials as the online version. Participants were indifferent between the two options in the first 25 trials $(M=50.26, S D=15.02), t(38)=.11, p=.916, d=.02,95 \%$ CI $[-.30, .34]$ and 40 trials $(M=48.33, S D=15.63), t(38)=-.67, p=.509, d=-.11,95 \%$ CI $[-.43, .21]$. Nonetheless, these findings suggest that people tend to tolerate doing nothing to avoid effort as they complete more trials. Lastly, as in Studies 10 and 11, social desirability was not significantly correlated with Stroop choices, $r=.00,95 \%$ CI $[-.32, .32], p=.977$.

\section{Meta-Analysis: Effort Choices, Trial Analyses, and Individual Differences}

The proportion of effort choices across all studies are on Figure 2. To verify the robustness of our findings, we ran a random-effects meta-analysis and confirmed that overall, participants did not choose to avoid effort to do nothing and, interestingly, chose effort significantly more than chance, though the effect is small and not robust to different exclusion criteria, $d=.21,95 \%$ CI $[0, .41], z=1.98, p=.047 . .^{5}$ The forest plot is shown on Figure 3 .

We also tested how choices changed over the duration of the studies. Figure 4 shows the predicted probabilities of effort choices. We fit a three-level generalized linear mixed model with trials nested within person, and person nested within study, with a random slope and intercept for trial number. Results showed a meta-analytic odds ratio of 0.99 [95\% CI $0.99-1.00], \mathrm{p}=.013$,

\footnotetext{
${ }^{5}$ With some and no exclusions, results showed no preference for effort nor doing nothing. See the forest plots on Figure S4 (self-exclusion), Figure S5 (no exclusion), and Figure S6 (attention check exclusions).
} 
suggesting that participants were $1 \%$ less likely to choose effort with every additional trial. Although participants did not avoid effort overall, they increasingly chose to endure doing nothing to avoid effort as time went on, consistent with past work on effort avoidance.

Finally, we examined the relationship between individual differences and choices. Scatterplots aggregated across studies are shown in Figure 5. Random effects meta-analysis revealed that need for cognition was significantly associated with effort choices, $r=.16,95 \% \mathrm{CI}$ $[.08, .24], z=4.14, p<.001$, suggesting that people who enjoy effortful activities avoid effort less. Meaningfulness of effort was also significantly associated with effort choices, $r=.08,95 \% \mathrm{CI}$ $[.02, .14], z=2.58, p=.010$, suggesting that people who find effortful personally meaningful also avoid effort less. Note, however, that both associations are modest in size. Finally, boredom proneness was not associated with effort choices, $r=-.01,95 \%$ CI $[-.08, .07], z=-.20, p=.840$, perhaps because people prone to boredom are quick to find effort dull (Danckert, 2019), and thus readily become indifferent between doing nothing and using effort.

\section{Discussion}

People tend to avoid cognitive effort. Here, we consider whether people would still avoid effort if the alternative option was to do nothing. People find it difficult to do nothing and are sometimes willing to work hard to escape it. Does the aversiveness of effort have precedence over the aversiveness of doing nothing? Using binary choice tasks, participants chose between an effortful task and doing nothing. We find that people do not necessarily avoid effort when the alternative was to do nothing and sometimes even avoided doing nothing in favor of using effort. Together, our findings demonstrate that doing nothing can be just as aversive - and sometimes even more aversive - than exerting cognitive effort. 
Comparing choices between effort and doing nothing provides insight into possible drives that motivate decisions to use or withhold effort. We found that people preferred high- but not low-demand tasks over doing nothing (Studies 1-6). This suggests that people do not necessarily prefer to do anything when faced with doing nothing. Instead, people may prefer only tasks that require some effort, seemingly suggesting that effort is sometimes valuable. The low demand tasks (add-1) may be so simple that it is "next to doing nothing" and thus people were indifferent. Additionally, although we expected that watching auto-completing Stroop trials would have been more stimulating than doing absolutely nothing and thus increased participants' willingness to choose it, we observed the contrary. People preferred to complete the Stroop task rather than watch it (Studies 8 and 9). We wonder if this result is due to the attenuated agency from watching the auto-completing Stroop trials. Past research has shown that people feel agency when they exert effort (Bussche et al., 2020; Osiurak et al., 2013). Even so, we found that people did not avoid effort and sometimes even preferred it when they preferred to count images instead of passively viewing them (Studies 10 and 11). This suggests that our findings that people sometimes prefer effort over doing nothing is not simply due to the lack of agency or desire for visual stimulation.

\section{When and Why do People Prefer Effort?}

People may have sometimes approached effort to avoid doing nothing because doing nothing provided little information. Information is valuable; it functions as a reward, and people are even willing to exert effort to gain information (Devine \& Otto, 2021; Marvin \& Shohamy, 2016). Even though the effortful option did not provide a lot of information, it still offered more than doing nothing. This is in line with recent theorizing suggesting that boredom promotes information-seeking (Agrawal et al., 2021; Danckert, 2019; Geana et al., 2016; Klapp, 1986). 
That is, boredom may "stop" people from avoiding effort by signaling that the task provides insufficient information. Interestingly, however, participants knew that the effortful option would not provide any rewards. This means that exerting effort would not have provided them with any new information. They knew from the beginning of the experiment what the expected value of the effortful option was. Yet, they still did not avoid it.

Even so, participants were more willing to tolerate doing nothing to avoid effort as the study progressed. We wonder if this is due to participants gradually learning that the effort task provided no new information, thus becoming duller as time went on. Although it did not do so by providing new information, it may have given participants the opportunity to update their mental models. People do not only make prediction errors about the world but also about themselves (Friston, 2013; Sun \& Firestone, 2020). As such, engaging in the effortful task, in which participants could mentally anticipate and track their own errors, may have generated high prediction errors. At the start of the studies, participants may have been motivated to avoid boredom by sampling from the effort task and thus exploring something mentally stimulating. Effort choices, that is, may have been initially driven by the desire to explore, thereby allowing participants to update mental models to help reduce long-term prediction errors.

From a cost-benefit perspective, choosing effort to avoid doing nothing may have been partly due to the very few opportunity costs of effort in our studies. When valuable alternative options are present, expending effort feels more aversive (Kurzban et al., 2013). Conversely, when valueless alternatives are present, using effort feels less aversive. Here, our alternative task intentionally minimizes the opportunity costs of choosing the high-effort option (i.e., they are foregoing the benefits of doing nothing), and therein likely reduces the averseness of effort. Likewise, it is also likely that having an effortful option as the only alternative to doing nothing 
reduced the aversiveness of doing nothing, given the effortful option was also aversive. This is in line with past work that finds that doing nothing in a room with many options for engagement was more aversive than sitting in a room without those options (Struk et al., 2020), suggesting that perhaps boredom may motivate the recruitment of effort due to its reduction of effort opportunity costs. Perhaps if our effortful tasks were more fatiguing and performance benefited from rest (Agrawal et al., 2021), the opportunity costs associated with effort would increase to reflect the value of rest, leading to an increased preference for doing nothing.

The law of least effort suggests that cognitive effort is avoided when all else is equal. It may initially appear that we keep all else equal aside for effort in several of our studies. In Studies 8 and 9, we presented the exact same Stroop stimuli with the only difference being that participants provided a response while in the other condition the computer provided the response. In Studies 10 and 11, both options presented the exact same stimuli, with the only difference being that in one of them we tasked participants with counting. However, it is difficult to control for all the inherent differences (aside from effort) between doing nothing and exerting effort. Specifically, doing nothing differs from exerting effort not only in terms of effort but also in terms of evoking boredom. And boredom independently shapes choices about what tasks to do, with boredom being costly, just like effort. Effort and boredom, that is, are independent predictors of task choice, and both states can be evoked by exerting effort and doing nothing. Doing nothing, for example, can be effortless and not boring (e.g., taking a break from exercising) and completing demanding tasks can be effortful and boring (e.g., working as a cashier). Thus, we interpret our findings as showing that boredom and effort are two variables that each shape choice, and that the disutility that comes with boredom is equal to and sometimes overshadows that of effort. 


\section{Alternative Explanations and Limitations}

There are a few possible alternative explanations to our findings. First, participants may have chosen effort instead of doing nothing because of demand characteristics. Because they were getting paid, doing nothing might have evoked cognitive dissonance, and they may have felt obliged to exert effort to reduce this dissonance. However, we believe this to be unlikely. In Studies 10 and 11, we manipulated payment and found no difference in effort choices. If choices were guided by feelings of obligation to choose effort because of payment, we would expect that a payment that was $50 \%$ higher might increase such felt obligation and increase effort choices in turn. We failed to find evidence for this, though we note that perhaps even higher payment would be needed for such moderation of choices. This is a limitation that should be more fully explored in future research.

Additionally, in Studies 10-12, we found that social desirability scores were not closely associated with effort choices. If people chose high effort options because of demand characteristics, those people who act in especially socially desirable ways should be even more likely to make such choices. However, we found no such association across 3 studies, further suggesting that choices were not strongly shaped by demand characteristics. Finally, in all studies we attempted to lower demand characteristics by informing participants that they should feel free to choose either option. In Studies 10-12, we additionally mentioned explicitly that both options provide us with equally valuable data, are perfectly valid, and that they should not feel that we expect them to prefer any one option over another and that both options are equally acceptable. Together, this suggests that although there is still the possibility that demand characteristics affected choices, we did not find evidence in support of it. 
Another alternative explanation is that participants may not have shown effort avoidance because doing nothing evoked negative thoughts, which could also be effortful in and of itself to regulate. However, we believe it is unlikely that choices were motivated by negative thoughts from doing nothing. In Studies 10-12, participants reported how sad or upset they felt while doing nothing and the effortful task. We found no difference in two of the three studies. Moreover, participants reported feeling less frustrated and working less hard while doing nothing than the effortful task, suggesting that even when participants experience greater negative thoughts while doing nothing, it does not evoke greater unpleasantness nor required much work to regulate.

Our studies are not without limitations. First, our do-nothing option was boring, and it might have induced spillover effects of boredom on subsequent choices. Unintentionally inducing boredom may have increased noisy decision-making (Yakobi \& Danckert, 2021). Second, conducting our studies online is particularly threatening to internal validity because participants could have easily escaped doing nothing by, for example, going on their phone. However, this lack of control should work against our predictions in so far that it makes it easier for participants to do something while they are supposed to do nothing. Similarly, participants who found effort and boredom especially aversive may have self-excluded themselves from the studies (by failing the attention checks). Due to COVID-19 pandemic restrictions, we were only able to conduct one study in the laboratory with a small sample size $(n=39)$ and a failed boredom manipulation (difference in boredom between options: $p=.053$ ). For most of our studies, we chose not to use difficult attention checks that could have motivated participants to attend to the screen because it would have defeated the purpose of the do-nothing option, doing nothing. This was why we used aggressive exclusion criteria, but it also suggests the need to replicate more of 
our findings in a controlled laboratory environment. Even so, when we included all participants including those who failed the attention checks and admitted to doing something else during the study - we still did not find evidence that people preferred doing nothing over effort. Third, we only ran our experiments in Western, Educated, Industrialized, Rich, and Democratic societies and would need to test this across other populations to be able to make statements about universal, pan-human tendencies (Henrich et al., 2010).

\section{Conclusion}

We have a fundamental drive to avoid effort. There has been a long-standing assumption that effort is costly. But when we reduce effort too much, when we are doing nothing, that is another powerful drive that motivates us into action. Only recently has research started to acknowledge the role of boredom in effort-based decision making. Although much work is needed to fully characterize how people allocate effort, here we introduce an important boundary condition: doing nothing. Does the disutility of doing nothing supersede the costs of cognitive effort? We demonstrate that effort is not avoided (and sometimes even preferred) when contrasted with doing nothing, indicating that doing nothing is just as - if not more - costly than exerting cognitive effort, at least under some conditions. 


\section{References}

Bench, S. W., \& Lench, H. C. (2013). On the function of boredom. Behavioral Sciences, 3(3), 459-472. https://doi.org/10.3390/bs3030459

Bench, S. W., \& Lench, H. C. (2019). Boredom as a seeking state: Boredom prompts the pursuit of novel (even negative) experiences. Emotion, 19(2), 242-254. https://doi.org/10.1037/emo0000433

Bussche, E. V. den, Alves, M., Murray, Y. P. J., \& Hughes, G. (2020). The effect of cognitive effort on the sense of agency. PLOS ONE, 15(8), e0236809. https://doi.org/10.1371/journal.pone.0236809

Boylan, J., Seli, P., Scholer, A. A., \& Danckert, J. (2021). Boredom in the COVID-19 pandemic: Trait boredom proneness, the desire to act, and rule-breaking. Personality and Individual Differences, 171, 110387. https://doi.org/10.1016/j.paid.2020.110387

Brodeur, M. B., Guérard, K., \& Bouras, M. (2014). Bank of Standardized Stimuli (BOSS) phase II: 930 new normative photos. PLOS ONE, 9(9), e106953. https://doi.org/10.1371/journal.pone.0106953

Cacioppo, J. T., \& Petty, R. E. (1982). The need for cognition. Journal of Personality and Social Psychology, 42(1), 116-131. https://doi.org/10.1037/0022-3514.42.1.116

Cameron, C. D., Hutcherson, C. A., Ferguson, A. M., Scheffer, J. A., Hadjiandreou, E., \& Inzlicht, M. (2019). Empathy is hard work: People choose to avoid empathy because of its cognitive costs. Journal of Experimental Psychology: General, 148(6), 962-976. https://doi.org/10.1037/xge0000595

Campbell, A. V., Chung, J. M., \& Inzlicht, M. (2022, June 26). Meaningfulness of effort: Deriving purpose from really trying. https://doi.org/10.31234/osf.io/sg3aw 
Celniker, J. B., Gregory, A., Koo, H. J., Piff, P. K., Ditto, P. H., \& Shariff, A. F. (2022). The moralization of effort. Journal of Experimental Psychology: General. https://doi.org/10.1037/xge0001259

Clay, G., Mlynski, C., Korb, F. M., Goschke, T., \& Job, V. (2022). Rewarding cognitive effort increases the intrinsic value of mental labor. Proceedings of the National Academy of Sciences, 119(5), e2111785119. https://doi.org/10.1073/pnas.2111785119

Cohen, J. D., McClure, S. M., \& Yu, A. J. (2007). Should I stay or should I go? How the human brain manages the trade-off between exploitation and exploration. Philosophical Transactions of the Royal Society B: Biological Sciences, 362(1481), 933-942. https://doi.org/10.1098/rstb.2007.2098

Dal Mas, D. E., \& Wittmann, B. C. (2017). Avoiding boredom: Caudate and insula activity reflects boredom-elicited purchase bias. Cortex, 92, 57-69. https://doi.org/10.1016/j.cortex.2017.03.008

Danckert, J. (2019). Boredom: Managing the delicate balance between exploration and exploitation. In J. R. Velasco (Ed.), Boredom is in Your Mind: A Shared PsychologicalPhilosophical Approach (pp. 37-53). Springer Verlag.

Devine, S., \& Otto, A. R. (2021). Making progress on the effort paradox: Progress information moderates cognitive demand avoidance. Proceedings of the Annual Meeting of the Cognitive Science Society, 43(43). https://escholarship.org/uc/item/58f4476f

Dunn, T. L., Inzlicht, M., \& Risko, E. F. (2019). Anticipating cognitive effort: Roles of perceived error-likelihood and time demands. Psychological Research, 83(5), 10331056. https://doi.org/10.1007/s00426-017-0943-x

Eastwood, J. D., Frischen, A., Fenske, M. J., \& Smilek, D. (2012). The unengaged mind: 
Defining boredom in terms of attention. Perspectives on Psychological Science, 7(5), 482-495. https://doi.org/10.1177/1745691612456044

Eyal, P., David, R., Andrew, G., Zak, E., \& Ekaterina, D. (2021). Data quality of platforms and panels for online behavioral research. Behavior Research Methods. https://doi.org/10.3758/s13428-021-01694-3

Faul, F., Erdfelder, E., Lang, A.-G., \& Buchner, A. (2007). G*Power 3: A flexible statistical power analysis program for the social, behavioral, and biomedical sciences. Behavior Research Methods, 39(2), 175-191. https://doi.org/10.3758/bf03193146

Ferguson, A. M., Cameron, C. D., \& Inzlicht, M. (2020). Motivational effects on empathic choices. Journal of Experimental Social Psychology, 90, 104010. https://doi.org/10.1016/j.jesp.2020.104010

Friston, K. (2013). Active inference and free energy. Behavioral and brain sciences, 36(3), 212. https://doi.org/10.1017/s0140525x12002142

Garavan, H., Ross, T. J., Li, S. J., \& Stein, E. A. (2000). A parametric manipulation of central executive functioning. Cerebral Cortex, 10(6), 585-592. https://doi.org/10.1093/cercor/10.6.585

Geana, A., Daw, N., Wilson, R. C., \& Cohen, J. D. (2016). Boredom, information-seeking and exploration. Proceedings of the 38th Annual Conference of the Cognitive Science Society, 6.

He, J., van de Vijver, F. J. R., Dominguez Espinosa, A., Abubakar, A., Dimitrova, R., Adams, B. G., Aydinli, A., Atitsogbe, K., Alonso-Arbiol, I., Bobowik, M., Fischer, R., Jordanov, V., Mastrotheodoros, S., Neto, F., Ponizovsky, Y. J., Reb, J., Sim, S., Sovet, L., Stefenel, D., ... Villieux, A. (2015). Socially desirable responding: Enhancement and denial in 20 
countries. Cross-Cultural Research, 49(3), 227-249.

https://doi.org/10.1177/1069397114552781

Henrich, J., Heine, S. J., \& Norenzayan, A. (2010). The weirdest people in the world? Behavioral and Brain Sciences, 33(2-3), 61-83. https://doi.org/10.1017/S0140525X0999152X

Hull, C. L. (1943). Principles of behavior: An introduction to behavior theory. AppletonCentury.

Inzlicht, M., Shenhav, A., \& Olivola, C. Y. (2018). The effort paradox: Effort is both costly and valued. Trends in Cognitive Sciences, 22(4), 337-349. https://doi.org/10.1016/j.tics.2018.01.007

Kahneman, D. (1973). Attention and effort. Englewood Cliffs, N.J: Prentice-Hall. Kool, W., \& Botvinick, M. (2014). A labor/leisure tradeoff in cognitive control. Journal of Experimental Psychology. General, 143(1), 131-141. https://doi.org/10.1037/a0031048

Kool, W., \& Botvinick, M. (2018). Mental labour. Nature Human Behaviour, 2(12), 899-908. https://doi.org/10.1038/s41562-018-0401-9

Kool, W., McGuire, J. T., Rosen, Z. B., \& Botvinick, M. M. (2010). Decision making and the avoidance of cognitive demand. Journal of Experimental Psychology. General, 139(4), 665-682. https://doi.org/10.1037/a0020198

Kurzban, R., Duckworth, A., Kable, J. W., \& Myers, J. (2013). An opportunity cost model of subjective effort and task performance. The Behavioral and Brain Sciences, 36(6), 10.1017/S0140525X12003196. https://doi.org/10.1017/S0140525X12003196 Leuba, C. (1955). Toward some integration of learning theories: The concept of optimal simulation. Psychological Reports, 1(1), 27-33. https://doi.org/10.2466/pr0.1955.1.g.27 
Marvin, C. B., \& Shohamy, D. (2016). Curiosity and reward: Valence predicts choice and information prediction errors enhance learning. Journal of Experimental Psychology: General, 145(3), 266-272. https://doi.org/10.1037/xge0000140

Milyavskaya, M., Galla, B. M., Inzlicht, M., \& Duckworth, A. L. (2021). More effort, less fatigue: the role of interest in increasing effort and reducing mental fatigue. Frontiers in Psychology, 12, 755858. https://doi.org/10.3389/fpsyg.2021.755858

Milyavskaya, M., Inzlicht, M., Johnson, T., \& Larson, M. J. (2019). Reward sensitivity following boredom and cognitive effort: A high-powered neurophysiological investigation. Neuropsychologia, 123, 159-168. https://doi.org/10.1016/j.neuropsychologia.2018.03.033

Nederkoorn, C., Vancleef, L., Wilkenhöner, A., Claes, L., \& Havermans, R. C. (2016). Selfinflicted pain out of boredom. Psychiatry Research, 237, 127-132. https://doi.org/10.1016/j.psychres.2016.01.063

Klapp, O. E. (1986). Overload and boredom: Essays on the quality of life in the information society. Greenwood Publishing Group Inc.

Petitet, P., Attaallah, B., Manohar, S. G., \& Husain, M. (2021). The computational cost of active information sampling before decision-making under uncertainty. Nature Human Behaviour, 5(7), 935-946. https://doi.org/10.1038/s41562-021-01116-6

Pfattheicher, S., Lazarević, L. B., Westgate, E. C., \& Schindler, S. (2020). On the relation of boredom and sadistic aggression. Journal of Personality and Social Psychology. https://doi.org/10.1037/pspi0000335

Raffaelli, Q., Mills, C., \& Christoff, K. (2018). The knowns and unknowns of boredom: A 
review of the literature. Experimental Brain Research, 236(9), 2451-2462. https://doi.org/10.1007/s00221-017-4922-7

Schouppe, N., Ridderinkhof, K. R., Verguts, T., \& Notebaert, W. (2014). Context-specific control and context selection in conflict tasks. Acta Psychologica, 146, 63-66. https://doi.org/10.1016/j.actpsy.2013.11.010

Seiler, J. P.-H., Dan, O., Tüscher, O., Loewenstein, Y., \& Rumpel, S. (2022). Experienced entropy drives choice behavior in a boring decision-making task. Scientific Reports, 12(1), 3162. https://doi.org/10.1038/s41598-022-06861-w

Shenhav, A., Musslick, S., Lieder, F., Kool, W., Griffiths, T. L., Cohen, J. D., \& Botvinick, M. M. (2017). Toward a rational and mechanistic account of mental effort. Annual Review of Neuroscience, 40, 99-124. https://doi.org/10.1146/annurev-neuro-072116-031526

Struk, A. A., Carriere, J. S. A., Cheyne, J. A., \& Danckert, J. (2017). A short boredom proneness scale: Development and psychometric properties. Assessment, 24(3), 346-359. https://doi.org/10.1177/1073191115609996

Struk, A. A., Scholer, A. A., Danckert, J., \& Seli, P. (2020). Rich environments, dull experiences: how environment can exacerbate the effect of constraint on the experience of boredom. Cognition and Emotion, 34(7), 1517-1523

Sun, Z., \& Firestone, C. (2020). The dark room problem. Trends in Cognitive Sciences, 24(5), 346-348. https://doi.org/10.1016/j.tics.2020.02.006

Tam, K. Y. Y., van Tilburg, W. A. P., Chan, C. S., Igou, E. R., \& Lau, H. (2021). Attention drifting in and out: The boredom feedback model. Personality and Social Psychology Review, 25(3), 251-272. https://doi.org/10.1177/10888683211010297

Westgate, E. C., \& Wilson, T. D. (2018). Boring thoughts and bored minds: The MAC model of 
boredom and cognitive engagement. Psychological Review, 125(5), 689-713. https://doi.org/10.1037/rev0000097

Wilson, T. D., Reinhard, D. A., Westgate, E. C., Gilbert, D. T., Ellerbeck, N., Hahn, C., Brown, C. L., \& Shaked, A. (2014). Just think: The challenges of the disengaged mind. Science (New York, N.Y.), 345(6192), 75. https://doi.org/10.1126/science.1250830

Wolff, W., Martarelli, C. S., Schüler, J., \& Bieleke, M. (2020). High boredom proneness and low trait self-control impair adherence to social distancing guidelines during the covid-19 pandemic. International Journal of Environmental Research and Public Health, 17(15), 5420. https://doi.org/10.3390/ijerph17155420

Wu, R., Ferguson, A. M., \& Inzlicht, M. (2022, May 23). Do humans prefer cognitive effort over doing nothing? Retrieved from osf.io/s4wv9

Vogel, T. A., Savelson, Z. M., Otto, A. R., \& Roy, M. (2020). Forced choices reveal a trade-off between cognitive effort and physical pain. ELife, 9, e59410. https://doi.org/10.7554/eLife.59410

Yakobi, O., \& Danckert, J. (2021). Boredom proneness is associated with noisy decisionmaking, not risk-taking. Experimental Brain Research, 239(6), 1807-1825. https://doi.org/10.1007/s00221-021-06098-5

Zipf, G. K. (1949). Human behavior and the principle of least effort. Addison-Wesley Press. 


\section{Figure 1}

\section{Task Schematic}

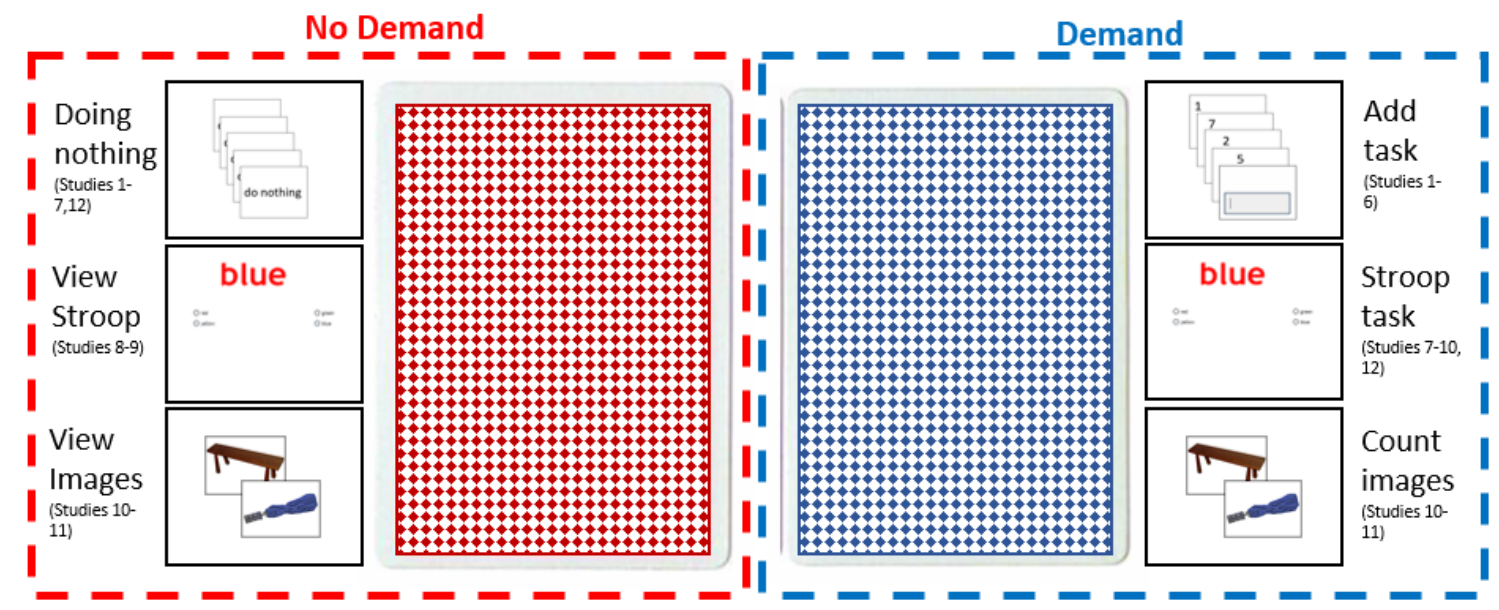

Note. In Studies 1 to 6, participants chose a deck and were required to do either the add task (one trial per choice) or were presented with a blank screen. In Study 7 and 12, participants were required to do either the Stroop task or were presented with a blank screen. In Studies 8 and 9, participants were required to do either the Stroop task or were presented with the Stroop task automatically completing itself. In Studies 10 and 11, participants chose to keep a running tally of two image counts or to passively view the same series of images. Images were taken from the Bank of Standardized Stimuli (Brodeur et al., 2014). 
Table 1

Descriptive Statistics of Phenomenology Across Twelve Studies

\begin{tabular}{|c|c|c|c|c|c|c|c|c|c|c|}
\hline \multirow{3}{*}{$\begin{array}{l}\text { Study } \\
\text { Name }\end{array}$} & \multicolumn{5}{|c|}{ Demand Rating } & \multicolumn{5}{|c|}{ Boredom Rating } \\
\hline & \multicolumn{2}{|c|}{$\begin{array}{l}\text { Demand } \\
\text { Deck }\end{array}$} & \multicolumn{2}{|c|}{$\begin{array}{l}\text { Do-Nothing } \\
\text { Deck }\end{array}$} & \multirow{2}{*}{$\begin{array}{c}\text { Effect } \\
\text { Sizes } \\
(d)\end{array}$} & \multicolumn{2}{|c|}{$\begin{array}{l}\text { Demand } \\
\text { Deck }\end{array}$} & \multicolumn{2}{|c|}{$\begin{array}{l}\text { Do-Nothing } \\
\text { Deck }\end{array}$} & \multirow{2}{*}{$\begin{array}{c}\text { Effect } \\
\text { Sizes } \\
\text { (d) }\end{array}$} \\
\hline & $M$ & $S D$ & $M$ & $S D$ & & $M$ & $S D$ & $M$ & $S D$ & \\
\hline Study 1 & 4.65 & 1.73 & 0.54 & 0.79 & 2.21 & 2.15 & 1.64 & 4.01 & 2.25 & -0.72 \\
\hline Study 2 & 4.21 & 1.82 & 0.65 & 0.83 & 2.05 & 1.76 & 1.47 & 3.78 & 2.12 & -0.94 \\
\hline Study 3 & 3.09 & 1.88 & 0.81 & 1.51 & 1.05 & 1.86 & 1.86 & 4.63 & 2.26 & -1.01 \\
\hline Study 4 & 3.41 & 1.59 & 1.39 & 1.71 & 0.93 & 2.46 & 2.13 & 5.29 & 1.99 & -1.08 \\
\hline Study 5 & 3.61 & 1.81 & 1.14 & 1.57 & 1.09 & 1.67 & 1.58 & 4.68 & 2.37 & -1.18 \\
\hline Study 6 & 4.12 & 1.52 & 0.37 & 0.71 & 2.24 & 1.80 & 1.59 & 3.87 & 2.21 & -0.93 \\
\hline Study 7 & 2.93 & 1.80 & 0.80 & 1.30 & 1.10 & 1.71 & 1.81 & 4.42 & 1.99 & -0.95 \\
\hline Study 8 & 3.23 & 2.20 & 0.98 & 1.18 & 1.25 & 2.41 & 1.59 & 4.51 & 2.20 & -0.92 \\
\hline Study 9 & 3.58 & 1.93 & 2.00 & 2.11 & 0.89 & 2.48 & 1.72 & 3.43 & 2.10 & -0.50 \\
\hline Study 10 & 2.42 & 1.82 & 0.77 & 1.48 & 1.00 & 2.60 & 1.91 & 3.82 & 2.58 & -0.54 \\
\hline Study 11 & 3.04 & 2.05 & 0.71 & 1.32 & 1.35 & 3.11 & 2.06 & 4.31 & 4.48 & -0.53 \\
\hline Study 12 & 1.84 & 1.71 & 1.23 & 1.40 & 0.47 & 3.79 & 2.14 & 4.35 & 2.22 & -0.32 \\
\hline
\end{tabular}

Note. Effect sizes represent Cohen's d from paired t-tests. All $p s<.001$, except in Study 12:

difference in demand, $p=.006$; and difference in boredom, $p=.053$. 
Table 2

Demand Choices Across Twelve Studies.

\begin{tabular}{cccccccccc}
\hline Study & \multicolumn{2}{c}{ Deck Type } & \multirow{2}{*}{ Trials } & $n$ & \multicolumn{5}{c}{ Effort Choices } \\
\cline { 2 - 7 } Name & Demand & Do-nothing & & & $M \%$ & $S D \%$ & $t$ & $d * 95 \%$ CI \\
\hline Study 1 & Add-3 & Do-Nothing & 40 & 63 & 59.64 & 19.92 & 3.84 & $.48^{* * *}$ & {$[.22, .75]$} \\
Study 2 & Add-3 & Do-Nothing & 80 & 63 & 58.25 & 20.28 & 3.23 & $.41^{* *}$ & {$[.15, .67]$} \\
Study 3 & Add-1 & Do-Nothing & 25 & 123 & 50.02 & 21.54 & 0.11 & .00 & {$[-.17, .19]$} \\
Study 4 & Add-1 & Do-Nothing & 40 & 62 & 49.15 & 22.59 & -0.30 & -.04 & {$[-.29, .21]$} \\
Study 5 & Add-1 & Do-Nothing & 40 & 146 & 45.57 & 21.81 & -2.46 & $-.20^{*}$ & {$[-.37,-.04]$} \\
Study 6 & Add-Variety & Do-Nothing & 40 & 105 & 55.24 & 22.41 & 2.40 & $.23^{*}$ & {$[.04, .43]$} \\
Study 7 & Stroop & Do-Nothing & 40 & 83 & 54.07 & 20.55 & 1.80 & .20 & {$[-.02, .42]$} \\
Study 8 & Stroop & Stroop Vid & 40 & 124 & 63.37 & 18.48 & 8.06 & $.72^{* * *}$ & {$[.53, .92]$} \\
Study 9 & Stroop & Stroop Vid & 100 & 90 & 67.30 & 23.35 & 7.03 & $.74^{* * *}$ & {$[.51, .98]$} \\
Study 10 & Count & View & 25 & 166 & 59.16 & 23.79 & 4.96 & $.38^{* * *}$ & {$[.23, .54]$} \\
Study 11 & Count & View & 40 & 161 & 51.02 & 25.74 & 0.51 & .04 & {$[-.12, .19]$} \\
Study 12 & Stroop & Do-Nothing & 100 & 39 & 40.05 & 18.72 & -3.32 & $-.53^{* *}$ & {$[-.88,-.20]$} \\
\hline
\end{tabular}

Note. Effect sizes represented with Cohen's $d$ from one-sample t-tests (two-tailed) with a test

value of $50 \% .{ }^{*} p<.05,{ }^{* *} p<.01,{ }^{* * *} p<.001$ 
Figure 2

Distribution of Effort Choices Across Twelve Studies

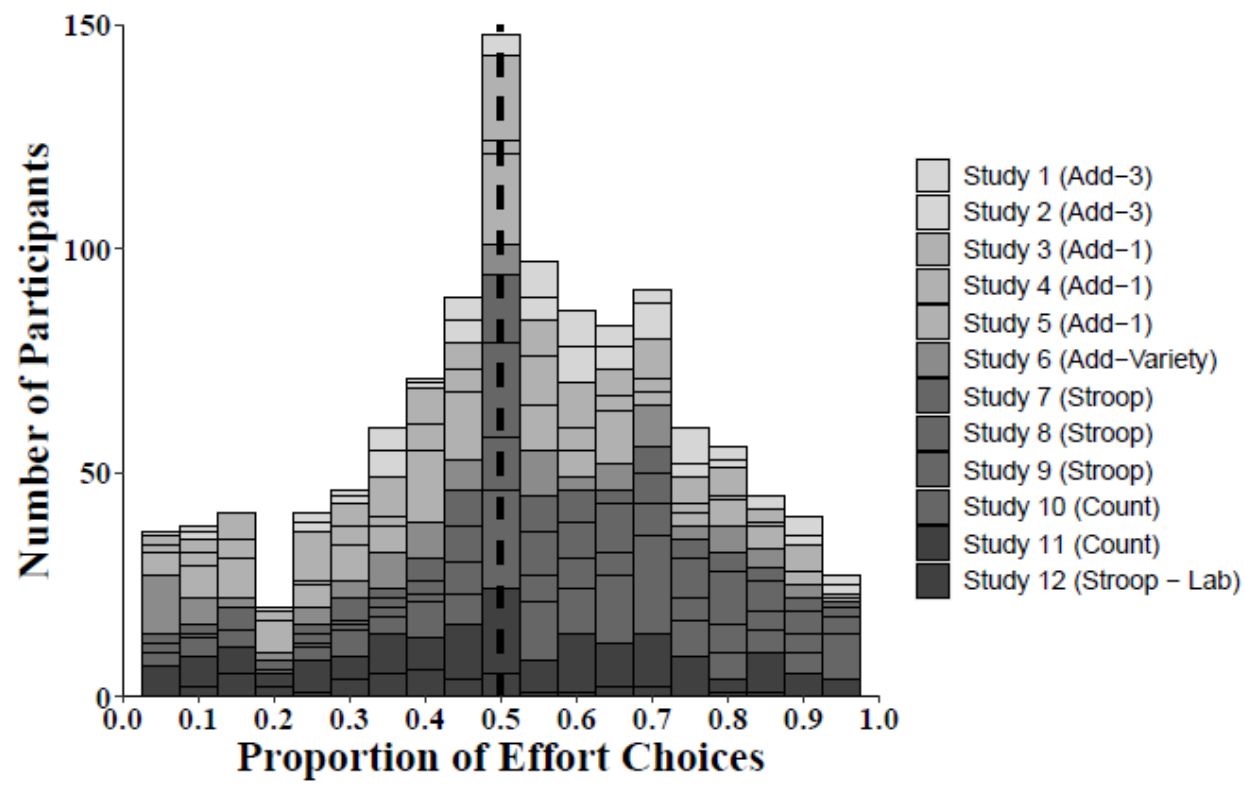




\section{Figure 3}

Forest Plot of Demand vs. Do-Nothing Choices Across Twelve Studies

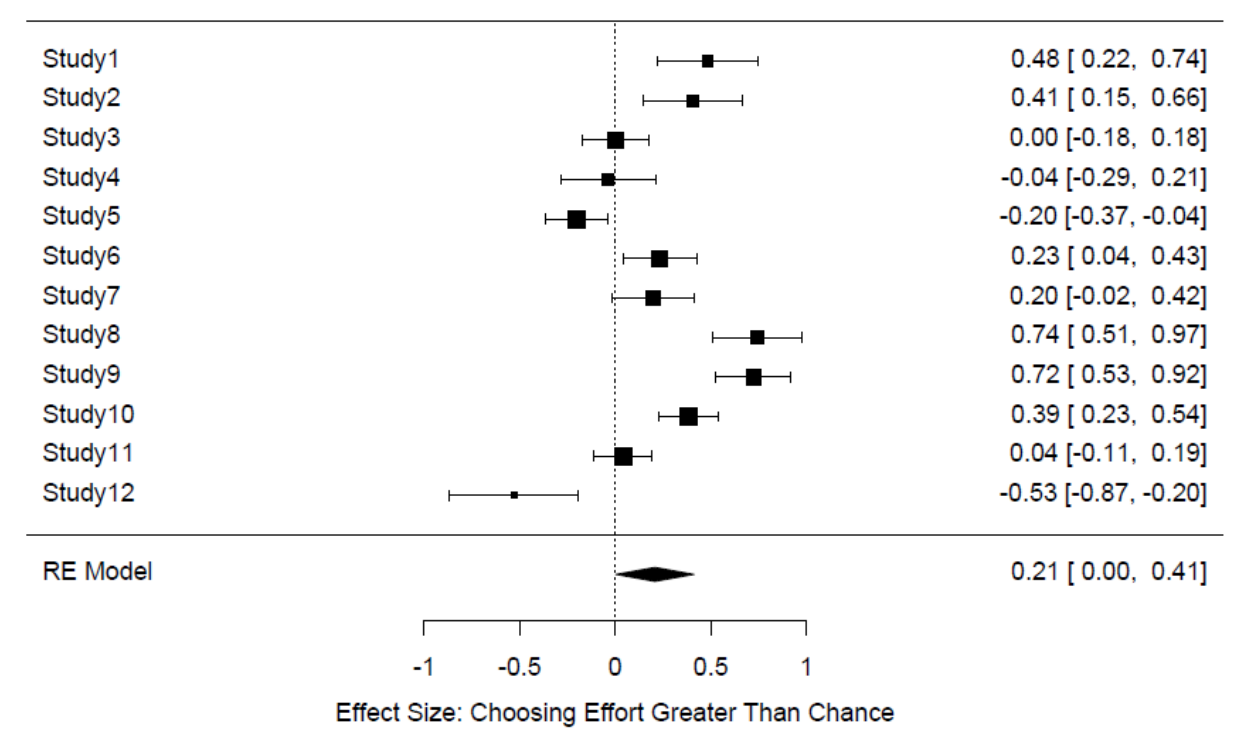

Note. Error bars represent 95\% confidence intervals. Effect sizes represent Cohen's d from onesample t-tests comparing the proportion of effort choices to $50 \%$. 


\section{Figure 4}

Predicted Probabilities of Effort Choices Across Trials from Twelve Studies.

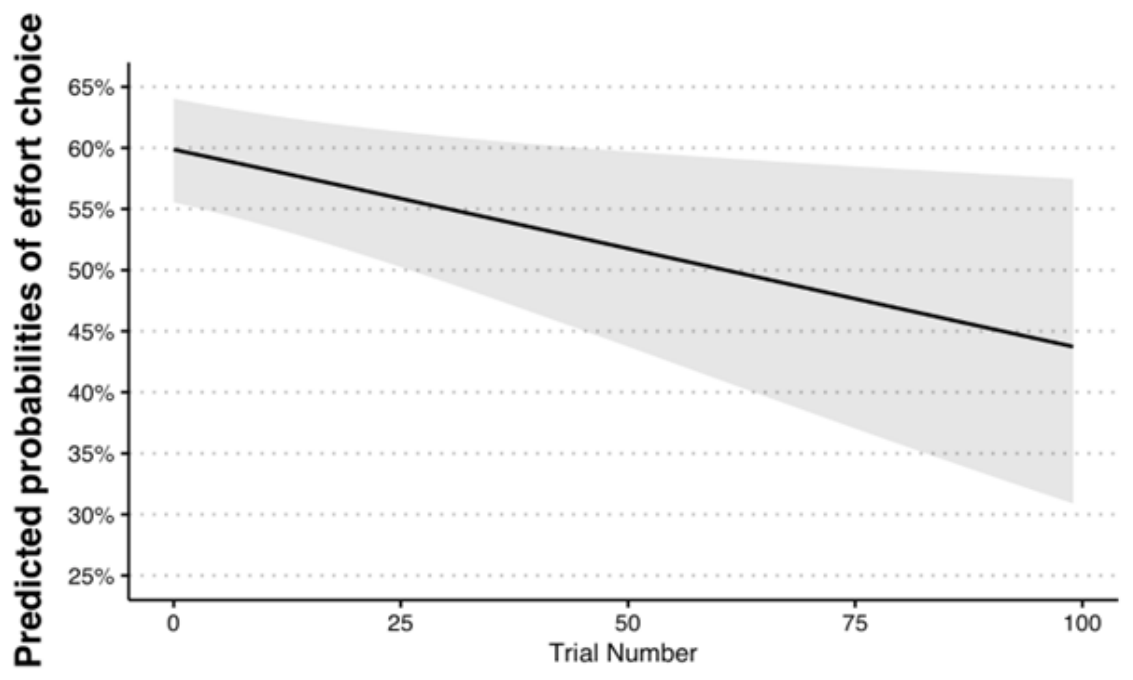




\section{Figure 5}

Scatterplots Showing the Relationship Between Individual Differences and Effort Choices

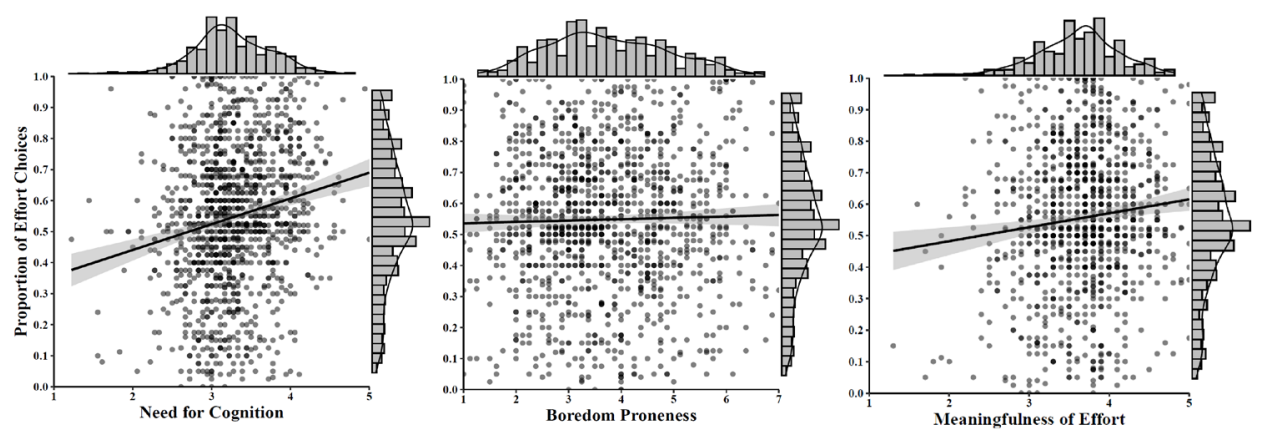

Note. Correlation between individual differences (need for cognition: $n=1225$; boredom

proneness: $n=1225$; meaningfulness of effort: $n=1040$ ) and effort choices aggregated across studies. The sample size is lower for meaningfulness of effort because that was not included in Studies 3 and 4. 